\title{
DOCTORATES CONFERRED IN 1958
}

The following are among those who received doctorates in the mathematical sciences and related subjects from universities in the United States and Canada during 1958. In each case when available, the university, the month in which the degree was conferred, minor subjects (other than mathematics), and title of the dissertation are given.

Note that there is included at the end of these Notes a list of doctorates conferred in 1957 but not included last year.

R. D. M. Accola, Harvard University, The bilinear relation on open Riemann surfaces.

M. S. Ahmed, University of California, Berkeley, January, On a locally most powerful similar test for the independence of two Poisson variables.

Yousef Alavi, Michigan State University of Agriculture and Applied Science, December, Expansions of parabolic wave and potential functions.

W. A. Al-Salam, Duke University, June, minor in Physics, The Bessel polynomials and some related functions.

Frederick Andrus, University of Florida, June, minor in Physics, Partially ordered ideal preserving groups.

W. D. L. Appling, University of Texas, May, minor in Business Administration, Concerning integrals.

H. J. Arnold, Princeton University, June, Permutation support for multivariate techniques.

E. F. Assmus, Jr., Harvard University, On the homology of local rings.

B. J. Attebery, University of Missouri, August, Some asymptotic properties of a maximum likehood estimator.

A. K. Aziz, University of Maryland, June, On higher order boundary value problems for hyperbolic partial differential equations in two or three variables.

G. G. Bach, McGill University, May, Three-body forces in hypernuclei.

J. L. Bailey, Michigan State University of Agriculture and Applied Science, March, A thermoelastic problem in the half-space.

R. E. Bargmann, University of North Carolina, June, minor in Psychology, $A$ study of independence and dependence in multivariate normal analysis.

C. W. Barnes, University of North Carolina, August, Polynomials associated with second order recursion sequences of integers. 
L. E. Baum, Harvard University, Derivations in commutative semisimple Banach algebras.

S. D. Baxter, University of Toronto, November, Numerical methods for the solution of hyperbolic differential equations with the aid of an electronic computer.

John Bender, Rutgers, The State University, June, On p-valent starlike functions and their partial sums.

J. A. Blankfield, University of Illinois, June, minor in Astronomy, Approximations to Einstein's equations of general relativity.

R. J. Blattner, University of Chicago, December, Group representation and operator rings.

Joseph Blum, George Washington University, February, Banach space functions and matrix summability methods.

E. W. Bold, St. Louis University, June, On partitions into unit and prime summands.

J. H. Bramble, University of Maryland, January, Reflection principles for solutions of equations in elasticity.

G. E. Bredon, Harvard University, Some theorems on transformation groups.

William Browder, Princeton University, June, Homology of loop spaces.

Morton Brown, University of Wisconsin, August, Continuous collections of higher dimensional hereditarily indecomposable continua.

R. W. Brown, Oregon State College, June, Solution of a nonlinear equation arising in a discontinuous control problem.

M. R. Bryson, Iowa State College of Agriculture and Mechanic Arts, August, Analysis of farm and home development benchmark survey results and associated statistical problems.

Roger Chalkley, University of Cincinnati, June, On the homogeneous second order quadratic differential field of characteristic zero.

Jacob Chassan, George Washington University, February, Probability and the state of mind.

Y. S. Chow, University of Illinois, February, minor in Physics, The theory of martingales in an S-finite measure space indexed by directed sets.

D. J. Christensen, University of Washington, June, Free products of $\alpha$-distributive Boolean algebras.

P. T. Church, University of Michigan, August, Some results in cluster set theory.

C. W. Clark, University of Washington, August, On the asymptotic distribution of the eigenvalues for the lower part of the Schrodinger operator spectrum. 
P. J. Cohen, University of Chicago, June, Topics in the theory of uniqueness of trigonometrical series.

W. W. Comfort, II, University of Washington, June, Šlov boundaries induced by certain Banach algebras.

James Conlan, University of Maryland, June, The Cauchy problem and the mixed boundary value problem for a non-linear hyperbolic partial differential equation in two independent variables.

Ellen Correl, Purdue University, January, Topologies for fields.

G. E. Cross, University of British Columbia, October, minor in Philosophy, On the uniqueness of multiple trigonometric series.

H. B. Curtis, Jr., Rice Institute, May, Properties of functions which uniformize certain classes of simply connected Riemann surfaces.

D. W. Dean, University of Illinois, June, minor in Philosophy, Projections in certain continuous function spaces.

M. H. DeGroot, University of Chicago, March, Unbiased sequential estimation of a probability.

J. H. DeHardt, University of Missouri, June, Uniform convergence of empiric integrals.

E. J. Delate, University of Buffalo, February, The power of a statistical test based on the mean successive difference.

T. L. Demen, St. Louis University, June, minor in Physics, $A n$ introductory analysis of complex functions by their four-dimensional representation.

D. G. Dickson, Columbia University, April, Expansions in series of solutions of linear difference-differential and infinite order differential equations with constant coefficients.

E. L. Diamond, University of North Carolina, June, Asymptotic power and independence of certain classes of tests on categorical data.

R. T. Dillon, University of Oregon, June, Invariant mappings between partially ordered vector spaces.

N. R. Draper, University of North Carolina, June, Rotable designs of second and third order in three or more dimensions.

J. B. Eades, Jr., Virginia Polytechnic Institute, June, An approximate solution for a cone-cylinder in axially symmetric transonic flow.

J. G. Elliott, Michigan State University of Agriculture and Applied Science, December, Characterizations of the symmetric difference and the structure of Stone algebras.

E. E. Enochs, University of Notre Dame, August, Primary reduced groups.

G. F. Feeman, Lehigh University, October, Characterizations of Riemann $N$-spheres. 
Harry Ferguson, University of Pittsburgh, June, An investigation of the stability of Chandrasekhar's solution of the Heisenberg spectral equation of homogeneous isotropic turbulence.

Ben Fitzpatrick, Jr., University of Texas, May, Operator matrices and systems of linear partial differential equations.

H. P. Flatt, Rice Institute, May, Infinitely differentiable solutions of ordinary differential equations.

S. R. Foguel, Yale University, June, Studies in spectral operators and the basis problem.

D. J. Foulis, Tulane University of Louisiana, August, Involution semigroups.

D. W. Fox, University of Maryland, June, The solution and Huygens' principle for a singular Cauchy problem.

Harry Furstenberg, Princeton University, June, Prediction theory.

A. E. Garrett, Virginia Polytechnic Institute, June, Estimation problems connected with stochastic processes.

J. J. Gart, Virginia Polytechnic Institute, June, Some problems in statistical inference.

R. H. Geeslin, Yale University, June, A class of diffusion equations.

S. M. Genensky, Brown University, June, Plane strain in a network of slightly extensible cords.

E. J. Gilbert, University of California, Berkeley, January, The identifiability problem for functions of finite Markov chains.

R. C. Gilbert, University of California, Los Angeles, June, Generalized resolvents of a symmetric operator.

R. P. Gilbert, Carnegie Institute of Technology, June, Singularities of three-dimensional harmonic functions.

Seymour Goldberg, University of California, Los Angeles, June, Linear operators and their conjugates.

J. M. Gonzalez-Fernandez, Northwestern University, June, Integrability of trigonometric series and Laplace-Stieltjes transforms.

Hugh Gordon, Columbia University, April, Topological Riesz spaces and $L_{p}$ spaces in relation to generalized integrals.

C. D. Gorman, Yale University, June, Brownian motion processes in Lie groups.

J. F. Gray, University of Notre Dame, August, Diagonal forms of prime degrees.

Leon Greenberg, Yale University, June, A class of compact 3-dimensional manifolds with constant negative curvature.

M. H. Greenberger, Harvard University, Computer simulation of the United States social economy.

Bernard Greenspan, Rutgers, The State University, June, $A$ bound 
for the order of the zero dimensional components of a system of algebraic difference equations.

J. J. Greever, III, University of Virginia, June, Fixed points of finite groups of transformations.

J. W. Greiner, University of Florida, February, minor in Education, Problem on Pellian equations.

J. H. Griesmer, Princeton University, June, On extreme games.

A. L. Gropen, Duke University, September, minor in Philosophy, Special homeomorphisms in the functional space $C\left(X, I_{2 n+1}\right)$.

Leonard Gross, University of Chicago, June, Integration and nonlinear transformation in Hilbert space.

Donald Guthrie, Jr., Stanford University, June, Bayes acceptance sampling procedures for large lots.

D. L. Guy, Washington University, January, Weighted p-norms and Hankel transforms.

R. M. Haber, Ohio State University, December, Term rank of 0, 1 matrices.

S. M. Harmon, University of California, Los Angeles, June, Regular covering surfaces of Riemann surfaces.

Bernard Harris, Stanford University, June, Determining bounds on integrals with applications to cataloging problems.

W. A. Harris, Jr., University of Minnesota, June, minor in Mechanics and Materials, $A$ boundary value problem for a system of ordinary linear differential equations involving powers of a parameter.

Robert Heller, Jr., University of Texas, August, minor in Business Administration, Some convergence theorems for continued fractions.

Leo Hellerman, Yale University, June, Minimal sets on a circle.

Michael Herschorn, McGill University, May, Some properties of Carathéodory solution of $X^{1}=f(t, x)$.

G. A. Heuer, University of Minnesota, June, Algebraic extensions of Banach algebras.

John Hilzman, Oregon State College, June, minor in Physics, $A p-$ plication of the Fréchet differential to the approximate solution of Volterra integral equations.

R. P. Holten, University of California, Berkeley, September, Generalized Goursat problem for a hyperbolic system.

H. C. Howard, Carnegie Institute of Technology, June, Oscillation criteria for fourth-order linear differential equations.

W. L. Hoyt, University of Chicago, March, Some decomposition theorems on Abelian varieties.

R. P. Hunter, Louisiana State University and Agricultural and Mechanical College, August, On the semigroup structure of continua. 
H. L. Hunzeker, University of Michigan, August, Torsion of shafts of varying circular cross section.

O. J. Huval, Louisiana State University and Agricultural and Mechanical College, August, minor in Physics, On complementation of normal subgroups and on automorphisms of groups.

J.-P. Imhof, University of California, Berkeley, September, Contributions in the theory of mixed models for the analysis of variance.

A. M. Jaqua, University of California, Los Angeles, February, Topological loops.

Walter Jaunzemis, Illinois Institute of Technology, June, Transient thermal stresses in an elastic half-plane.

A. W. Jenkins, Jr., University of Virginia, June, On the singleparticle nature of the oxygen nucleus.

H. B. Jenkins, University of Southern California, June, Conservation laws and two-dimensional variational problems.

R. P. Jerrard, University of Michigan, June, Vibration of hollow anisotropic thick-walled cylinders.

H. T. Jones, Brown University, June, An application of the theory of linear operators in Hilbert space to a problem in potential theory.

R. K. Juberg, University of Minnesota, June, minor in Physics, On the Dirichlet problem for the equation $\Delta^{2} U+U_{t}=0$.

W. M. Kahan, University of Toronto, May, minor in Physics, Causs-Seidel methods of solving large systems of linear equations.

R. B. Kellogg, University of Chicago, December, Hyperbolic equations with multiple characteristics.

R. B. Kelman, University of California, Berkeley, June, The asymptotic behavior of the real solutions of certain differential-functional systems.

Harry Kesten, Cornell University, June, Symmetric random walks on groups.

J. E. Kimber, Jr., Massachusetts Institute of Technology, June, minor in Physics, Obstructions to H-structures.

E. K. Kinney, University of Illinois, February, minor in Philosophy, Integral equations in representation theory of functions of a complex variable.

P. G. Kirmser, University of Minnesota, June, minor in Chemical Engineering, Initial and boundary value problems associated with the partial differential equation $U_{x x x}+U_{t}=0$.

M. I. Knopp, University of Illinois, October, minor in Philosophy, On the construction of certain automorphic forms on non-negative dimension.

U. R. Kodres, Iowa State College of Agriculture and Mechanic 
Arts, November, Characterization of functions whose second differences approach zero.

George Kolettis, Jr., University of Chicago, December, On primary Abelian groups.

L. H. Koopmans, University of California, Berkeley, June, Asymptotic rate of discrimination for Markov processes.

K. S. Kretschmer, Carnegie Institute of Technology, June, Linear programming in locally convex spaces and its use in analysis.

R. R. Kuebler, Jr., University of North Carolina, August, minor in Public Health, On the construction of a class of error-correcting binary signaling codes.

K. W. Kwun, University of Michigan, June, Characterization of the n-sphere through decompositions, and related topics.

G. A. Ladner, University of Kansas, On solvability of factorizable groups.

P. J. Laufer, McGill University, May, The structure of left $H$-star algebras.

J. W. Layman, University of Virginia, June, Contribution of twoand three-nucleon potentials to binding energy per nucleon using and extended source nucleon.

C.-W. Lee, Illinois Institute of Technology, June, A study of thick plates under tangential loads applied on the faces.

Milton Lees, University of California, Berkeley, January, $A p$ proximate solutions of parabolic and hyperbolic partial differential equations.

Stanislaw Leja, Cornell University, September, Inversion of a function with the kernel $1 /\left[1+(x-y)^{2}\right]\left[c^{2}+(x-y)^{2}\right]$.

H. S. Leonard, Jr., Harvard University, $A$ generalization of a theorem of Blichfeldt.

E. L. Lima, University of Chicago, December, Duality and Postnikov invariants.

G. O. Losey, University of Michigan, February, Group rings and dimension subgroups.

R. D. Low, Iowa State College of Agriculture and Mechanic Arts, June, Symmetrical deformation of circular cylinders possessing transverse cylindrical aeolotropy.

Albert Madansky, University of Chicago, June, Identification and estimation in laten class.

Gabriel Margulies, Indiana University, September, minor in Mechanics, The differential invariants of local regular surface mappings. 
A. W. Marshall, University of Washington, August, On the growth of stochastic processes.

L. B. Martin, Jr., Harvard University, Approximation by ratios of integers chosen from a limited range-applications to the gear train problem.

P. E. Martin, Harvard University, Extremal metrics for a class of variational problems related to extremal length.

W. S. Martindale, III, University of Pennsylvania, February, The commutativity of certain classes of rings.

Eben Matlis, University of Chicago, June, Injective modules.

A. A. McCauley, Catholic University of America, June, minor in Physics, A study of linear systems of differential equations.

P. E. McDougle, University of Virginia, June, Quasi-compact and related mappings.

H. E. McKean, Purdue University, August, Utilization of chromosomes in quantitative inheritance.

A. W. McKinney, University of California, Berkeley, September, On the limiting behaviour of the real solutions of certain first order nonlinear ordinary differential systems.

R. L. McKinney, University of Washington, August, Positive bases for linear spaces.

L. R. McMurray, University of Illinois, June, minor in Electrical Engineering, Harmonic hypervariables.

R. K. Meany, University of Wisconsin, June, minor in Physics, Differential equations for sequences.

G. H. Meisters, Iowa State College of Agriculture and Mechanic Arts, June, Almost periodic movements in uniform spaces.

E. P. Merkes, Northwestern University, June, Some properties of univalent $S$-fractions.

R. J. Mihalek, Illinois Institute of Technology, June, Modularity relations in lattices.

K. L. Miller, Brown University, June, Wave propagation in ring springs under general loading.

R. G. Miller, Jr., Stanford University, June, A contribution to the theory of bulk queues.

G. J. Minty, University of Michigan, June, Integrability conditions for vector fields in Banach spaces.

W. D. Montgomery, Iowa State College of Agriculture and Mechanic Arts, August, Cleavage of inseparable field products.

Benjamin Muckenhoupt, University of Chicago, August, On certain singular integrals. 
T. W. Mullikin, Harvard University, Mixed Cauchy problems and $C_{0}$-semigroups.

E. A. Newburg, University of Illinois, June, minor in Electrical Engineering, Potential representations in integral equations.

D. J. Newman, Harvard University, Some aspects on polynomial approximation.

Edward Norman, Cornell University, September, Topics in discreet convolution transforms.

C. C. Oehring, University of Tennessee, December, Spectral theory of self-adjoint ordinary differential operators.

Kôtaro Oikawa, University of California, Los Angeles, June, On the stability of boundary components.

C. C. Oursler, Illinois Institute of Technology, June, Topics in the theory of matrices.

R. B. Paine, University of Washington, August, Fixed points of the circle group acting on a cohomology sphere.

Cristina Parel, University of Michigan, June, A matrix derivation of generalized least squares linear regression with all variables subject to error.

G. W. Patterson, University of Pennsylvania, June, Algebraic foundations of number-representation systems.

R. N. Pendergrass, Virginia Polytechnic Institute, June, The rank analysis of triple comparisons.

P. A. Penzo, University of Pittsburgh, June, A class of Norlund methods of finite order.

M. M. Phelps, Harvard University, The closed subalgebras of a commutative algebra over the real numbers.

R. R. Phelps, University of Washington, June, Subreflexive normed linear spaces.

J. M. Pike, Oregon State College, June, minor in Electronics and Communication, Mountain waves with neutral thermodynamic stability.

M. J. Poliferno, Yale University, June, Decision algorithms for some functional calculi with modality.

R. P. Polivka, University of Illinois, June, minor in Electrical Engineering, $A$ generalization of singular integral equations with complex kernels.

M. B. Pour-El, Radcliffe College, Computable functionals.

E. J. Putzer, University of Minnesota, December, minor in Physics, $A$ method for investigation of the properties of solutions of the equation $x^{\prime \prime}+f(x, t) x^{\prime}+g(x, t)=\phi(t)$.

W. A. Raab, Iowa State College of Agriculture and Mechanic Arts, July, Virtual mass of a deformable body.

A. L. Rabenstein, Jr., Massachusetts Institute of Technology, 
June, minor in Electrical Engineering, Asymptotic solutions of a class of fourth order differential equations.

F. A. Raymond, University of Michigan, June, Poincaré duality in homology manifolds.

B. E. Rhoades, Lehigh University, October, Hausdorff summability methods.

R. W. Richardson, Jr., University of Michigan, August, Actions of the group $S O$ together (3) and $S P$ together $(L)$ on the spheres $S^{4} S^{5}$.

L. H. Roberts, University of Florida, August, minor in Physics, Effect of an elliptic disk of orthotropic material on the stress distribution in an orthotropic plate.

G. S. Rogers, State University of Iowa, June, On statistics whose distributions depend upon a parameter.

B. A. Rojas, Iowa State College of Agriculture and Mechanic Arts, June, The analyses of groups of similar experiments.

S. M. Rosenzweig, Massachusetts Institute of Technology, September, minor in Physics, An analogy of Witt's theorem for modules over the ring of $p$-adic integers.

R. A. Ross, University of Toronto, May, minor in Physics, The waves produced by a submarine earthquake.

S. E. Ross, Harvard University, Variational solutions to optimal rocket trajectory problems.

O. S. Rothaus, Princeton University, June, Domains of positivity.

N. J. Rothman, Louisiana State University and Agricultural and Mechanical College, August, Homomorphisms and topological semigroups.

H. R. Rouse, Vanderbilt University, August, minor in Philosophy, Topological dynamics and a partly ordered set of topologies.

I. D. Ruggles, Iowa State College of Agriculture and Mechanic Arts, June, Minimum norm solution of a linear equation in Hilbert space in terms of solutions of related projected equations.

G. A. Salton, Harvard University, An automatic data processing system for public utility revenue accounting.

B. H. Sams, III, University of Illinois, June, minor in Physics, Finite groups of auto-morphisms of algebraic function fields.

W. G. Saunders, University of Pittsburgh, June, Uniformly continuous sets in metric spaces.

F. S. Scalora, University of Illinois, June, minor in Astronomy, Abstract martingale convergence theorems.

S. H. Schot, University of Maryland, June, Sonic limit singularities in the hodograph method.

D. S. Scott, Princeton University, June, Convergent sequences of complete theories. 
R. T. Seeley, Massachusetts Institute of Technology, September, Minor in Modern Language, Singular integrals on compact manifolds.

J. L. Selfridge, University of California, Los Angeles, August, On finite semigroups.

Eugene Sevin, Illinois Institute of Technology, June, On the elastic bending of columns due to dynamic axial forces including effects of axial inertia.

Robert Silverman, Ohio State University, December, $A$ metrization for power-sets and Cartesian products with applications to combinatorial analysis.

G. F. Simmons, Yale University, June, The fine structure of spectra in Banach algebras.

W. E. Smith, University of California, Los Angeles, August, On posteriori probabilities.

Louis Solomon, Harvard University, The representation of finite groups in algebraic number fields.

M. M. Stanisic, Illinois Institute of Technology, June, On the oscillation of 5-wing in supersonic flow.

Manuel Stein, Virginia Polytechnic Institute, June, Postbuckling behavior of rectangular plates.

M. C. Stippes, Virginia Polytechnic Institute, June, Some mixed and associated boundary value problems in the theory of thin plates.

D. G. S. Stockton, Brown University, June, Singular parabolic partial differential equations with time dependent coefficients.

K. R. Stromberg, University of Washington, June, Probabilities on a compact group.

David Sussman, McGill University, May, On certain subgroups of algebraic matrix groups at prime characteristic.

L. J. Teutonico, Brown University, June, Theory of the thermal breakaway of a pinned dislocation line with application to damping phenomena.

R. J. Thompson, Ohio State University, June, On essential absolute continuity for a transformation.

D. E. Thoro, University of Florida, August, minor in Philosophy, On the representation of integers by indefinite ternary quadratic forms.

E. O. Thorp, University of California, Los Angeles, June, Compact linear operators in normed linear spaces.

D. B. J. Tomiuk, Catholic University of America, June, minor in Physics and Philosophy, Lattice-complemented algebras.

R. N. Townsend, University of Illinois, October, minor in Physics, Boundary value distributions.

W. F. Trench, University of Pennsylvania, June, On an explicit method for the numerical solution of a Stefan problem. 
L. B. Treybig, University of Texas, May, minor in Physics, Concerning locally peripherally separable spaces.

D. A. Trumpler, Massachusetts Institute of Technology, June, minor in Physics, Periodic solution of an autonomous singular perturbation problem.

S. F. Tuan, University of California, Berkeley, September, Some calculations in the quantum mechanics of elementary particles.

D. H. Tucker, University of Texas, May, Absolutely continuous solutions of $U x y=f\left(x, y, u, u_{z}, u_{y}\right)$.

P. J. Villemure, University of Notre Dame, June, Collective singularities of families of analytic functions.

R. E. Walpole, Virginia Polytechnic Institute, June, Combined intra- and inter-block analysis for factorials in incomplete block designs.

P. C. Waterman, Brown University, June, Multiple scattering of waves.

H. J. Weinitschke, Massachusetts Institute of Technology, September, minor in Physics, Finite axisymmetrical bending and buckling of shallow spherical shells.

Daniel Weiser, Rice Institute, May, The behavior of functions harmonic and positive in an angular domain.

G. H. Weiss, University of Maryland, June, Thermodynamic properties of a disordered lattice.

L. R. Welch, California Institute of Technology, The rearrangement of functions and maximization of a convolution integral.

J. H. Wells, University of Texas, May, minor in Physics, Concerning the Hausdorff inclusion problem.

J. V. Whittaker, University of California, Los Angeles, June, Multiple transitive groups of transformations.

A. D. Wiggins, University of California, Berkeley, January, A statistical study of the mechanism of bacterial toxicity.

R. E. Wild, University of California, Los Angeles, June, On the number of lattice points in $x^{t}+y^{t}=n^{t / 2}$.

W. H. Williams, Iowa State College of Agriculture and Mechanic Arts, June, Unbiased regression estimators and their efficiencies.

Alan Wilson, Rice Institute, May, Collective singularities of families of analytic functions.

C.-H. Yang, University of Cincinnati, June, Integrability of trigonometric series with monotone decreasing coefficients.

Kapbyung Yoon, Syracuse University, Algebraic Laplacian and discrete boundary value problems in a simplical complex.

J. N. Younglove, University of Texas, August, minor in Physics, Concerning dense metric subspaces of certain non-metric spaces. 
George Zyskind, Iowa State College of Agriculture and Mechanic Arts, August, Structures in experimental designs.

The following doctorates were conferred in 1957, but were not included in the list in the preceding volume of this Bulletin (vol. 64, pp. 125-136):

R. D. Boswell, Jr., University of Georgia, June, Flows of isometries.

L. C. Butler, University of Illinois, June, $A n$ imbedding for locally product spaces.

W. E. Hartnett, University of Kansas, October, Upper and lower measures in a metric space and some applications to the theory of spread measure.

R. G. Heyneman, University of California, Berkeley, September, On uniqueness in abstract ergodic theory.

M. W. Hirsch, University of Chicago, December, Immersions of manifolds.

H. H. Johnson, University of California, Berkeley, September, Prolongations of differential systems.

D. V. Keller, University of California, Berkeley, September, $\mathrm{Nu}$ clear interactions of antiprotons.

George Pedrick, University of Kansas, October, Theory of reproducing kernels for Hilbert spaces of vector valued functions.

Barth Pollak, Princeton University, October, Quadratic arithmetic in quaternion algebras.

M. S. Rohatgi, University of California, Berkeley, September, I. Locally unbiased tests of composite hypotheses with $s$ constraints. II. Estimation of the location parameter based on certain order statistics. III. Distance estimates based on luminosity functions.

C. E. Watts, University of California, Berkeley, September, $A$ general cohomology theory of sheaves. 